\title{
Téoros
}

Revue de recherche en tourisme

\section{Le P'tit Train du Nord et ses successeurs}

\section{Normand Cazelais}

Volume 15, numéro 1, printemps 1996

Les Laurentides, quelles Laurentides?

URI : https://id.erudit.org/iderudit/1075052ar

DOI : https://doi.org/10.7202/1075052ar

Aller au sommaire du numéro

Éditeur(s)

Université du Québec à Montréal

ISSN

0712-8657 (imprimé)

1923-2705 (numérique)

Découvrir la revue

Citer cet article

Cazelais, N. (1996). Le P’tit Train du Nord et ses successeurs. Téoros, 15(1),

33-38. https://doi.org/10.7202/1075052ar d'utilisation que vous pouvez consulter en ligne.

https://apropos.erudit.org/fr/usagers/politique-dutilisation/ 


\title{
LE P'TIT TRAIN DU NORD ET SES SUCCESSEURS
}

\author{
Normand Cazelais
}

\section{Normand Cazelais \\ journaliste et géographe à Hydro-Québec.}

\section{Introduction}

En 1891, quinze ans après I'Inauguration de la vole ferrée rellant Saint-Jérơme à Montréál, le curé Labelle, le *rol du Nord, voyalt l'un de ses vleux rêves accompll: cette année-là, des ouvrlers posalent les premiers ralls de la ligne devant rejoindre MontLaurler. Enfn, ses chers Pays-d'en-Haut étalent ouverts à la colonisation. SI ce chemin de fer eut le succès escompté1. II servit davantage à attirer des touristes que des agriculteurs dans les Laurentides de Montréal.

Après avoir connu de longues années de glolre, notamment. auprès des skleurs, Jusqu'au début des annces 50 , le P'til. Train du Nord chanté par Féllx Leclerc connut un rapide déclin. devant la concurrence de l'automoblle pour laquelle fut réaménagće à plusleurs reprises la route 11 (devenue la 117 en 1972) et fut construlte à grands frals un peu plus tard la section dite panoramique de l'Autoroute des Laurentides. Au milleu des années 70, le Canadien Paciflque. à la fols proprlétalre de l'emprise et exploitant de la ligne. déposalt auprès de la Commlssion des transports du Canada ses premlères demandes d'abandon du transport des pas. sagers; ces demandes allalent être rapldement exaucées pulsque la compagnle cessa ce service vers la fin de la decennle, ouvrant la vole aux expériences fort temporalres de trains touristiques Jusquau 15 novembre 19812.

Par un curleux retour des choses, labandon des lialsons ferrovalires dans les Laurentides et la transformation subséquente de leurs emprlses en parcs linéaires articulés autours de plstes polywalentes donneront encore, vers la fin du XXe slècle, de nouvelles avenues au développement touristique de cette réglon qui, en apparence, compte parmi les plus performantes du Québec ${ }^{3}$.

\section{Une référence: la Route verte}

En JuIn 1995 eut Ileu le lancement ofliciel de la Route verte, prolet d'utinéraires cyclables inlégrés de 2500 kllomètres quil devratent couvrir le Québec d'lel dix ans, à partir d'accotements paves, de chaussées désignées et d"emprises ferrovlaires recyclées. Ce circult =vert*, pulsque son concept le veut respectueux de l'environnement, ballsé et sécuritaire. est une Inltlattve de Vélo Québec qui agira comme maître d'oeuvre du projet en collaboration avec le gouvernement du Québec et de nombreux partenaires publics et privés.

Un premier tronçon-pllote entre Montréal et Sherbrooke en sera lamorce. *La Route verte représente le moyen de concrétıser un grand rêve, celul d'un vérltable Québec cyclablé (...). partagé par blen des Québccols. En effet, l'appul vient de partout et laccuell dans l'opinion publique est extraordi- naire: (selon un sondage), $90 \%$ des Québécols se disent favorables au concept de la Route verte.-4

*Toutefols, pour avoir chez sol le plus long itinéralre cyclable du monde, le prix à payer est relativement élevé. On estime que les Investissements privés s'élèveront à quelque $80 \mathrm{mll}-$ llons \$. Heureusement, on prévolt des retombées économiques du même ordre pour la province, étant donné qu'll s'agit d'un prodult touristique facllement exportable qul suscitera un grand intérêt chez les amateurs de vélo des quatre colns du monde, lesquels seront tentés de s'y donner rendez-vous. Dans le sud de la province, on projette même de faire le raccordement avec les pistes cyclables américaines, $=5$

Un schéma préliminalire a été publlé à l'occasion du lancement de la Route verte qui met également de l'avant la mise en place graduelle de comité réglonaux ayant pour mandat de regrouper les intervenants du milleu et de favoriser une élaboration collective de tronçons réglonaux. SI l'Idée de base du schéma est la réalisation d'un *Itunéraire cyclable-. elle n'exclut pas d'autres vocations. *Compte tenu des prlorités réglonales ou des objectifs d'un gestlonnaire de sentler, rien n'empêche qu'une section de la piste solt également utilisable pour la marche, le ski ou même la motoneige. (...) D'un point de vue purement technique, la Route verte n'est pas une plste cyclable. C'est d"abord un itinéralre qui pourra parfols emprunter une vole ferrée abandonnée et recyclée ou encore des portions de routes tranquilles où le flot de circulation est faible. Litinéralre pourra même suivre une route plus importante dont le pavage des accotements assurera une melleure securité aux cyclistes. Cette approche d'itinéralre cyclable est tout à falt semblable à celul proposé par les grands itinéraires cyclotouristıques américains ou européens...6 6

En réglon, des segments ont précédé la nalssance de la Route verte. Maintenant que le gouvernement du Québec a racheté 40 kilomètres d'emprise entre Cabano et la frontlère du Nouveau-Brunswick, il ne manque que trols kilomètres à Rlviè re-du-Loup pour compléter une pliste de 120 kilomètres, le Sentier Rivière-du-Loup/T'émiscouata, qui s'étirera dans le Parc linéaire du Petit Témis Jusqu'à Edmunston. Certalns tronçons ont également été rachetés: par exemple, dans la Gatineau (d'une longueur de 62,4 kilomètres entre Low et Maniwaki) et dans le Bas-Saint-Laurent (266 kllomètres). Plusieurs autres sont dans le collimateur. sans que cela ne se situe toutefols, le déplore Vélo Québec, dans une approche aglobale et intégrées (au sud de Québec, entre Rlchmond et Charny, au Temlscamingue sur 134 kllomètres). alors que, par allleurs, le gouvernement du Québec négocle avec le $\mathrm{CN}$ et le $\mathrm{CP}$ pour le rachat en bloc d'une quinzaine de tronçons (environ 800 kllomètres au total) répartis un peu partout au Québec'7. 
La plste cyclable du Parc linéaire Le P'tut Train du Nord s'inscrit dans cette perspective.

\section{Le Parc linéaire Le P'tit Train du Nord}

Au début des années 80 , à la sulte de la disparition d'un service qul fut longtemps de premlère nécessité, un équipement Important, tant par sa tallle que par sa localisation, se trouvait libéré. Source de problèmes pour les uns, cette situation s'avéralt prometteuse pour d'autres. Outre évidemment la question du financement, le hic était qu'il n'y avalt guère de précédents au Québec en la matlère et que la classe polltique en général ne manlfestait pas un Intérêt débordant envers un projet voulant recycler une emprise ferrovlalre en un corridor récréotouristique de 200 kilomètres. Qul plus est, pour ajouter aux réticences, ce *parc linéalre* étalt destuné à accuellil r, exception falte de la motoneige, des activItés d'apparence peu prometteuses au plan commerclal (vélo. randonnéce pédestre, skI de fond). Sans compter bon nombre de riverains qui manifestaient ouvertement leur désir de s'approprler des portions de l'emprlse à la fols pour agrandir leurs proprlétés et pour préserver leur tranqulllité. Le Canadlen Paciflque, propriétalre de l'emprise, privlléglalt cette approche, on s'en doute.

Le projet prit plus de hult ans pour arriver à son terme. En 1987. l'idée falsalt déjầ l'objet de discussions à l'Association touristique des Laurentides (ATL) quil décida d'envoyer deux personnes dans le Midwest des États-Unis, notamment au Wisconsin et dans l'Illinols, pour étudler sur place quelques cas de parcs linéalres. Un peu partout, dans les mllleux cyclistes et de plein air mais aussi écologiques ou partisans d'autres solutions que les grandes infrastructures wen dur-, se manlfestèrent des appuls.

En 1989, l'ATL mandata la firme SOTAR pour réallser le plan directeur et une étude de faisabilité du parc IInéaire. Un comité de gestion, formé de représentants des ministères concernés et d'organismes réglonaux intéressés par le projet, en adopta le rapport final qui fut présenté au mllleu en 1991. La même année nalssalt la Coalition régionale de préservation de l'emprise du Canadien Pacifique et d'avancement du parc linéaire des Laurentides. Pour sa part, la Corporation de développement des Laurentides a assumé le secrétariat jusqu'à l'acquisition du corridor en 1994.

Les encadrés cl-contre résument les grandes étapes de l'évolution du projet et livrent les principales caractéristiques. tant physiques qu'organlsationnelles, du parc linéaire Le P'tit Train du Nord.

\section{PARC LIVÉAIRE LE P"TIT TRAIN DU NORD FICHE TECHNIOUE}

Localisation

Entre Salnt-Jérôme et Mont-Laurler.

\section{Dimensions}

200 kllomètres (longueur); de 24,5 à 30,5 mètres (largeur); 568 hectares (superflcle).

Caractérlstlques physiques (ouvrages et équipements) Revêtement en criblure de plerre; pente constante de $2 \%$; 14 ponts, 92 ponceaux, ballast et murs de soutènement.

Accès

90 croisements routlers.

\section{Environnement}

Forêt $(67 \%)$, urbain/seml-urbain (20\%), villéglature $(8 \%)$, agriculture (5\%): 41 kllomètres aux abords de lacs et de rivières.

Coûts d'acqulsittion

3,5 millions \$.

Coûts d'aménagement

3,5 millions $\$$.

\section{Proprlétaire}

Gouvernement du Québec (ministère des Affaires munlclpales).

\section{Gestlonnalres}

Les municipalités réglonales de comté Antolne-Labelle (45\% du trajet), La Rivlère-du-Nord (7\%), Les Laurentides $(38 \%)$, Les Pays-d'en-Haut (10\%).

\section{Partenalres}

Association touristique des Laurentides, CADC AntolneLabelle, GADC LAurentides/Pays-d'en-Haut, Chambres de commerce, Comité Intermunicipal de développement ćconomique de Labelle, Consell de la Culture des Laurentides, Consell régional des lolsirs des Laurentides, Corporation de développement des Laurentldes, Corporation des gares des Laurentides, Entreprises du secteur privé. Ministère de l’Environnement et de la Faune du Québec, Ministère du Tourlsme du Québec, Office de la Planiflcation et du Developpement du Québec, Tourisme Canada.

\section{Isages autorisés}

Été: vélo et marche.

Hiver: ski de fond de Salnt-Jérôme à Val-David, motonelge de Sainte-Agathe-des-Monts à Mont-Laurler.

(Source: Coalition du Parc linéaire Le P'tit Train du Nord 


\section{PARC LINÉAIRE LE P'TIT 'TRAIN DU NORD IIISTORIOLE}

1983:

Premlère demande d'abandon, par le Canadien Paciflque. du transport ferrovlaire de marchandises.

1984:

Dépốt des premlers prolets locaux de réutillsation de l'emprise à des fins de randonnée et de plein alr.

1986:

Inscription comme prolet prioritaire, dans le plan de développement touristique des Laurentides, de la prếservation de l"emprise et de sa transformation en parc linéalre: formation d'un premier comité.

\section{9-90:}

Abandon progressif du transport ferrovlaire entre SaintJérôme et Mont-Laurler.

\section{0:}

Réallsation du Plan directeur de développement et d'amenagement du Parc linéalre des Laurentides.

1991:

Enlèvement des ralls et des dormants: création de la Coalution du Parc linéalre des Laurentides qui négocie un accord pour exclure la démolition des ponts.

\section{2}

Location de l'emprise par le Consell régional des loisirs des Laurentides (CRL) et par I'Assoclation motonelge translaurentienne; embauche de patroulleurs par le CRL durant l'été pour observer les utillsateurs (autorisés ou non) de l'emprise devenue sentler polyvalent.

1994:

Achat de l'emprise par le gouvernement du Québec pour 3,5 millions \$; location du sentjer aux MRC de la région: réalisation des travaux d'aménagement par Gaz Métropolitain (enfoulssement de la conduite principale) et par les MRC: ouverture du sentier au ski de randonnée.

\section{$1995=96$ :}

Ouverture du sentler au vélo et poursuite des travaux d'aménagement (qui auront coûté 3.5 millions $\$$ aux municipalités, dont une contribution de $475000 \$$ par Gaz Métropolitaln et de 1.1 million \$ par Hydro-Québec dans le cadre de sa politique de mise en valeur environnementale).

Source: Coaltition du Pare Inéalre Le P'tut Train du Nord)

\section{Structures de gestion, vocation, achalandage el promotion}

Pour le gouvernement du Québec, le minlstère des Transports se chargea de l'acquisition de l'emprise au coût de trols milllons et demi de dollars et. sitôt la transaction falte, en délégua la gestlon au minlstère des Affalres municlpales.

Par la sulte, les quatre municipalités réglonales de comté (MRC) traversées par l'emprise, el conséquemment par le futur parc llnéraire réglonal (Antoine-Labelle, La Rlvlère-duNord, Les Laurentides, Les Pays-d'en-Haut), slgnèrent un ball de location de l'emprise de 60 ans renouvelable, les autorisant à agir à titre de gestionnaires officlels du parc Innéalre Le P'tit Train du Nord. Celles-cl formèrent une Coalition dont l'objectif est :d'assurer une harmonisation dans les différents champs d'activités: la gestion, la promotion, les communications, la sécurité, l'entretien, l'aménagement et le contrồle des activités et des événements* ${ }^{8}$.

Près de 2.6 mlllions \$ (dont 0.5 mlllion par Gaz Métropolitain pour l'enfouissement de sa conduite principale et un million par Hydro-Ouébec dans le cadre de sa politique de mise en valeur environnementale) ont été invesus jusqu'à présent d'un bout à l'autre du parc. La Coalition compte aménager les cinq derniers kilomètres, entre Prếvost et Pledmont, au printemps 1996.

Les activités aujourd'hul permises sont le vélo et la marche en été sur l'ensemble du circult et, en hlver, le skI de fond entre Saint-Jérôme et Val-David (sur une distance de 45 kilomètres) et la motonelge entre Salnte-Agathe-des-Monts et Mont-Laurier (sur 155 kilomètres). L'hiver, des organlsmes qualifies et des clubs de motonelge y assurent, sur leurs territoires respectifs, la sécurité et l'entretien des plstes alors qu'un système de patrouille à vélo sera Instauré à l'été 1996.

Selon les évaluations de la firme SOTAR en 1990, le potentiel de jours-utilisation du parc linéaire pour les quatre activités précitées s'inscrit dans une fourchette annuelle varlant de 800000 à 4,7 millions. Une comparalson avec ce qui se passe depuls plus de vingt ans aux Etats-Unls permet d'établir à 6000 jours-utilisateurs au kilomètre la moyenne annuelle de fréquentation d'un parc linéalre: «L'expérience vécue durant l'été 1995 nous permet de crolre que l'ob/ectif maximum d"achalandage, solt 1.2 mllllon de jours-utlllsateurs, sera atteint d'icl trols ans $z^{9}$

Tout cecl demandera des efforts de promotion. La Coalition publlera en avril 1996 la premlère éditıon du Gulde de services olliciel du parc. Encarté dans le Guide touristique de l'ATR. ce document de 16 pages comprendra des cartes et une description sommaire des services disponlbles au fil des kilomètres. Enfin, un évếnement d'envergure soullgnera, au printemps de la même année. l'inauguration officielle du parc linéare Le P'tit Train du Nord.

\section{Traits originaux, problèmes et perspectives}

La récupération d'un espace libéré de ses fonctions orlginales - qui en implique le recyclage à d'autres fins - cons- 


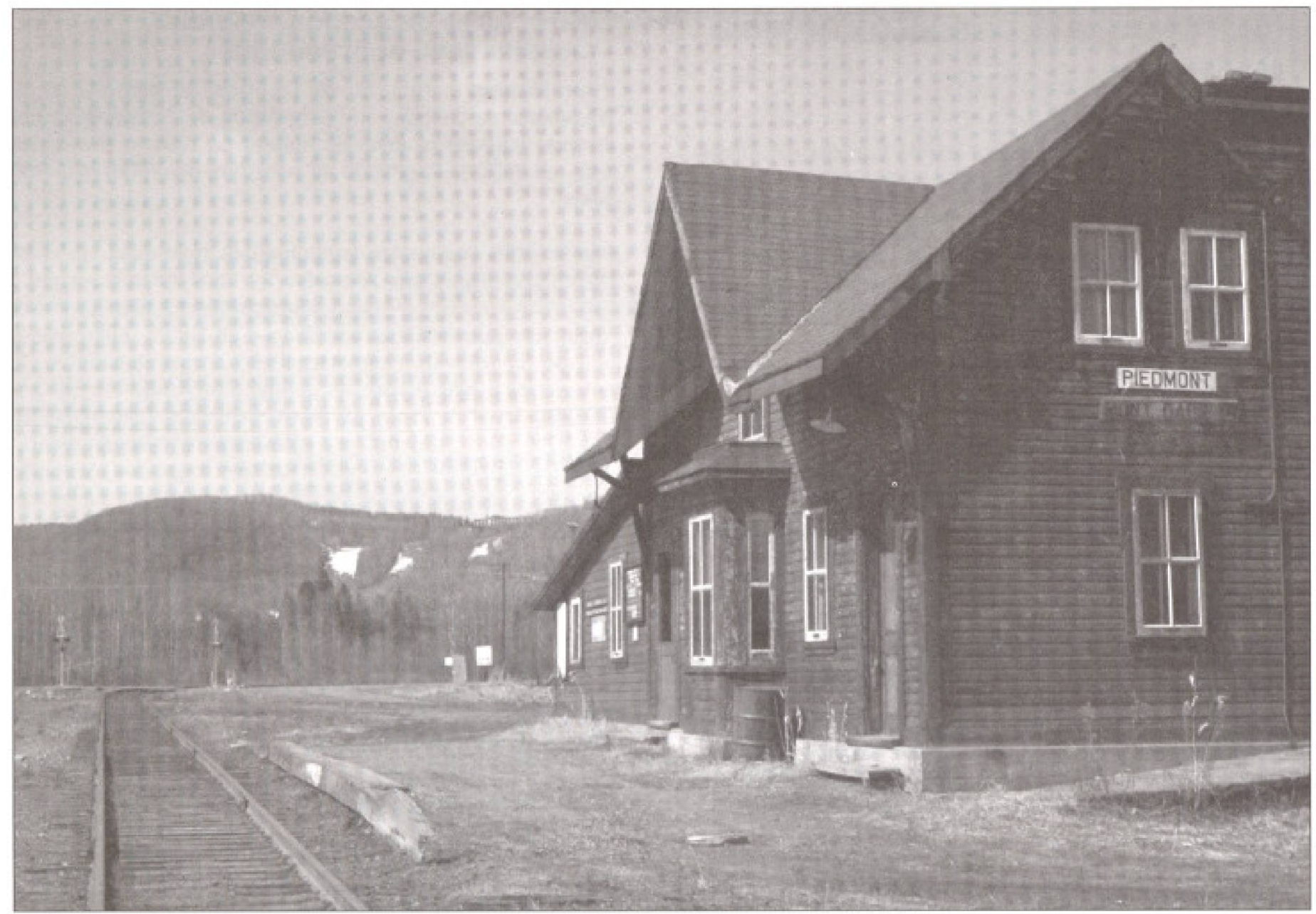

Ancienne gare de Piedmont

Litue l'élément distinctif d'un equipement comme le parc linéaire Le Ptut Train du Nord. En sol, l'acquisition de l'emprise, pour 3,5 millions de dollars. représente presque une aubalne: comblen en coutteralt-ll aujourd'hul pour acquérir. déboiser et défricher. dans un milieu aussi soumis à la spéculation fonclère que les Laurentides de Montréal, un corridor public de 568 hectares sur une longueur de 200 kllomètres?

Outre une évidente fillation avec l"histoire de la réglon et tout son patrimolne (l'essor économlque et touristique des Pays-d'en-Haut lié à la venue du chemin de fer), ce parc possède d'autres tralts caractéristiques. Il est:

- une source d'appel Identiflée non pas à un lieu fixe et déterm iné mals à un clrcult (ćest-ă-dire à un ensemble de points ou lieux interreliés) incitant. par son essence méme, ses utilisateurs à traverser une réglon en tout ou en partie et à en découvrir les attraits;

- à la fols un canal de fréquentation récréotouristique et un outıl de diffusion et d'éclatement de cette fréquentation. en ctant accessible sur l"ensemble de son parcours et. à l'Inverse, en donnant accès aux lleux et territoires quil lui sont contigus:

- un équlpement public géré, sur une base régionale el locale, a des fins collectives, exigeant dès l'orlgine la concertation tant de ses promoteurs que de ses bénéficlaires publics ou privés (corps publics, utillsateurs, commercants):

- un équipement polyvalent voué à divers usages (skl ou motoneige en hiver, marche et vélo le reste de l'année), impliquant, d'une part, des infrastructures dites légères et un minlmum d'aménagements à alouter à l'emprise préexistante et, d'autre part, un -zonage- et des règles d"utilisation précis pour éviter des conflits nulsibles à son dóveloppement et à son image:

- une Image, enfin, associée en général aux activités de plein air peu perturbatrices ${ }^{10}$. a la fréquentation d'espaces naturels ou perçus comme tels et à la mise en valeur de l'environnement, autant de valeurs fort prisées à l'heure actuelle.

Déjà, en sa brève existence, le parc linéaire a eu des effets structurels:

- en bordure de son parcours sont apparus des equipements de support (dépanneurs, boutlques de location et de réparation de vélos ou de skis de randonnée, gîtes du passant. ete.) dont le nombre et la variété sont appelés à croître dans un proche avenlr;

- une remise en valeur d"un passé encore tout proche falt surface, notamment par l'attention accordée au potentiel attractif et patrimonlal des quelques gares (huit au total) qui n'ont pas été démolles et qui subsistent, pour cer- 
taines, dans un état plus qu'Inqulétant11;

- se manlfeste une intégration spatlale de dlvers lleux jusqu'Icl séparés sinon isolés, comme c'est le cas avec les parcs de la Rivlère-du-Nord (à Prévost) et de la Doncaster (à Mont-Rolland) qul, par leurs llens directs avec I'anclenne emprise, ont été désenclavés et qui dolvent, par conséquent, a]uster leurs vocations respectives à une affluence plus considérable et différemment étalée dans le temps;

- de plus en plus apparalssent des forfaits autour d'activités pratiquées dans le parc.

Lévidente popularité du parc Iinéare Le Ptit Train du Nord lllustre le blen-fondé d’un tel concept qui, on le sait, a fait ses preuves allleurs, que ce solt aux Etats-Unis ou en Europe (en Autriche, en Allemagne et en Angleterre en particuller). Ce qul ne veut pas dire, pour autant que tous les écuells sont maîlrisés. A l'Instar du parc lul-même, devront être apportées des approches et solutions originales, particulièrement adaptées à son contexte.

- Le partage, durant les mols de nelge, entre le skI de fond au sud et la motonelge au nord ne fait pas l'unanimité. La llgne de partage, a la hauteur de Val-Davld, au coeur d'une dense villéglature, entre les usagers de l'une et l'autre de ces activités, constitue à cet effet une zone déllcate que la bifurcation actuelle ne dessert pas de façon optimale. L'image et, conséquemment, le développement à long terme du parc risquent d'en souffirir sl aucun correcuf n'est apporté.

Le plus dur a été accompli dans l'éllmination des activités Indés|rables: convalncre les amateurs de motocross et de véhlcules tout terrain que la plste ne convenalt à leurs acuvités ne fut pas une mince alralre: la mise à l'écart des adeptes de l'équitation et des chlens en lalsse est plus discutable et une réflexion devrait se poursuivre là-dessus. De toute façon, se pose la question de l'application des règlements édictés pour le parc: la Coalition (ou toute autorité quil lul succédera) aura à déterminer, dans un proche avenir, comment elle devra se falre et comment. Des approches mises de l'avant aux États-Unis pourralent être de valables sources dinspiration.

- Qul, en relève à la Coalition, assumera la gestion et l'entretlen du parc? Les munlcipalités, quil ne sont guère équipées pour une telle opératıon? Des clubs d'usagers selon le modèle de l'Appalachlan Trall ou du Sierra Club? Lientreprise privée?

Celte question lève le couvercle du financement. En ces temps où les pouvoirs publics se retirent tant de l'administration directe que du financement d'équipements de ce type, où la recherche de commandites se révèle de plus en plus ardue. Il Importe de prévolr à long terme, sinon le parc rlsque de pérlcliter. Une entreprise, à savolr Gaz Métropolitain, a récemment manifesté son intentlon de supporter des interventions à caractère historique le long du parcours et à y associer son nom. Y en aura-t-ll d'autres, et à quelles conditions? Quelle sera l'implication des com= merçants? Toujours intéressés à entendre sonner leurs throlrs-calsses, ceux-ci ne manifestent pas le méme empressement à pulser dans leurs poches pour contribuer de leurs deniers à une entreprise quil ne leur appartient pas en propre ou sur laquelle Ils n'ont pas une autorité directe de gestion. Les convalncre ne fera pas tout seul, tout comme Il ne sera pas alsé d'assurer une diversiflcation adéquate des services commerciaux attenant au parc, tant au plan de leur nature qu'à celul de leur localisation.

La Coalition - qui n'a guère le cholx - jongle délà avec l'Idée d'imposer une tarification qui devra être imaginative et souple, en ralson de la longueur de la piste, de ses multiples accès et du nombre d'utillsateurs. Blen conçe et bien appllquéc. celle-cl aura toutefols l'avantage non seulement d'assurer des rentrées de fonds nécessaires à l'exploltation et à la maintenance du parc mais aussı de développer un sentiment d'appartenance et de solldarité chez les usagers.

- La propreté du parc et la protection de son environnement par chacun des usagers devra constituer une prlorité. Une active campagne de sensiblilsation sera nécessaire; des responsabilités à cet eflet devront être déléguées à des Intermédiaires (clubs, etc.), des panters à ordures devront ètre installés à des endroits stratégiques et surtout leur vidange devra ètre régulièrement assurée. Sl elles peuvent s'expliquer par le jeune âge du parc, les Insufflsances actuelles ne sauralent durer: celul-cl perdra toute sa force d'attraction.

- La vigllance des municipalités dans l'application de leurs réglementalions respectives aux abords du parc sera de mise. Plus ce dernler aura du succès, plus se feront de pressions pour y Implanter toutes sortes de commerces, n'importe comment, Li'idéal seralt même de déboucher sur une certalne homogénéité dans la vision et les exlgences tant aménagistes qu'architecturales; or le nombre de municipalités concernếes et la longueur du parc rendent une telle approche fort difficile sinon impossible. Pourtant, II en va de la qualité du produit Louristique du parc et de son pouvolr attractif.

- Comme son nom le souligne, la personnalité profonde du parc est nourrie d'histolre. Mals, hors l'emprise et le ballast, rien ou presque ne subsiste de ce train: tout a été enlevé, rails, dormants et clous; quelques rares poteaux de signalisation tiennent encore, comme oublićs: même le tablier a été refalt, de la criblure de plerre a été épandue à sa surface et on a pelne à reconnaître çà et là des traces de l'ancien ouvrage: le ballast a souvent changé de gabarit; le parc ainsı beaucoup perdu de son âme. Inutlle, par ailleurs d'insister sur l'état des gares, II parle par lulmême. Le parc ne saurail malntenlr sa personnalité que sur le seul pouvolr évocateur d'un nom: l'histolre du train - du P'tit Train du Nord - devra revivre, et dans des manifestations concrètes (mise en valeur du patrimoine bâti existant, panncaux de rappel, reconstitutions, etc..). La mémoire a besoin de matériaux tangibles.

De même, il seralt fort intéressant de trouver, au long du circult, des panonceaux expliquant la géographle environnante (le relief et ses origines, le cycle des salsons et les transformations de la végétation, la faune observable, la vie des villages, la toponymle des alentours el ses sources, etc.): ces 
divers éléments appartlennent au corpus même du parc qul a repris, répétons-le, le relals d'une vole (l'emprise ferrovialre) et d'un moyen (le train) d'accès aux Laurentldes de Montréal. Il importe donc de les intégrer le plus intımement. possible à son produit. Dans cette perspectlve, la mise sur pled de visites guldées (et la formation conséquente des guides) seralt, comme en tout parc digne de ce nom, essentielle.

\section{Conclusion}

Le chemIn parcouru est certes considérable. Il en reste encore davantage à falre, ne seralt-ce que pour Implanter une structure de gestion et des modes de financement aptes à assurer le développement du parc à long terme. Il sera également essentiel, dans un horizon plus rapproché, de clrconscrire avec précision les retombées économlques et touristiques d'un tel équipement.

Comme une autoroute et une route, ce parc est un équipement linéaire: Il Importera d'évaluer son pouvolr réel de rétention de ses utillsateurs et conséquemment de sa force d'attraction et de démultiplication touristique. Et, au même tutré que sa IInéarité permet une Intégration réglonale du prodult touristique des Laurentides de Montréal, II seralt. hautement souhaitable de l'insérer lul-mème au sein d'un ensemble plus vaste tel que la Route verte.

\section{Bibliographie}

GALLANT, Brigitte, Le vélo roule sa bosse! Journal du minlstère des Transports du Québec, vol. 25, no 8, octobre 1995, Ouébec.

Les sentlers du 21e slècle - Planiflcation, deslgn et gestion des senters polywalents, tradustion et adaptation sous la direction de Jean-Françols Pronovost, en collaboration avec Marc Lusignan et Françolx Marcll, Montréal, Vélo Québec, 1995, 223 p.

La Route verte, Bulletin d'Information, Vélo Québec, vol. 1 no 2, décembre 1995, Montréal, 4 p.

Parc IInéalre Le P'th Train du Nord - Au coeur des Laurentldes, brochure de présentation, Munlcipalité réglonale de comté des Laurentudes, décembre 1995, non paginée.

Parc Inéalne Le P'LL Traln du Nord - Section MRC AntoineLabelle, dépllant d'Information (Incluant une carte), La Munlcipalité réglonale de comté Antolne-Labelle, MontLaurler, hlver 95/96.

Parc linéalre Le P'tu Train du Nord - Sectlon MRC de la RIvlère-du-Nord, dépliant d'Information (Incluant une carte), La Municlpalité réglonale de comté La Rıvlère-duNord, Salnt-Jérôme, hiver 95/96.

Parc Inealre Le P'tI Traln du Nord - Section MRC des Laurentides, dépllant d'information (incluant une carte), La Municipalité régionale de comté des Laurentides, Sainte-Agathe-des-Monts, hiver 95/96.

Panc llnéalre Le P'tlt Traln du Nord - Sectlon MRC des Paysd'en-Haut, dépliant d'information (Incluant une carte). La Municipalité réglonale de comté des Pays-d'en-Haut, Salnte-Adèle. hiver 95/96.

Notes

1 II fut même doublé, pendant plusieurs décennieg, par une autre vole ferróke, tachetute plus tard par le Canadien National, pour expedier le minerai des mines de kaolin de Salnt-Rémi-d"Amherst vers Saint-Jébome et Montréal en comprun-tant une partie de la vallée de la Rouge et le nord du comte d'Aracnteull (Weir, Arundel, Huberdeau, Lac-des-Seize-lles, Laurel, Montfort, Morin Heights. Saint-Sauveur-des-Monts).

2 Pour sa part. l'autre wole ferrés avait déjà dét abandonnée et plus tand démantelóe en 1962, après que le Canadien National y eüt cegsé toute activité en 1957.

3 Consulter à cet effet les rapports statistiques annuels de Tourisme Québec.

4 PRONovosT, Jean-François; directeur gënéral, Vẻlo Québec, in La Route verte, Bulletin d'information, Vélo Oućbec. wol. 1 no 2 , décembre 1995. Montréal. p. 1.

5 GALLANT, Brigitue, Le vélo roule sa bossef, Journal du ministére des Transports, vol. 25. no 8, octobme 1995, Ouébec

6. La Route verte, Bulletin d'information, Vélo Qućbec, vol, 1 no 2, décembre 1995, Montréal, p. 3.

7 Le moratoire, imposé par Duchece, sur la wente d'emprises ferroviaires par les compagnies de chemin de ler, prendra fo en mat 1996. Dans les mois qui précéderont, le gouvemement aura le chook de reporter la fin du moratoire. d'autoriser la vente à des intértüs privés ou de négocier avec ces compagntes pour un tachat en bloc.

8 Panc lindaine Le Ptit Train du Nord - Au coeur des Launentides, brochure de présentation, Municipalité régionale de comté des Laurentides. décembre 1995. non paginée.

9 lbidem.

10 Sauf pour la motoneige.

11 Dans ces certains cas, ces références deviennent paradoxales, telle cette Aubcrge do la Gare en ce qui lut Sainte-Marquerite-Station, la gare et toute trace matérielle pouvant en subsister ayant disparu depuis longtemps. 